\title{
Taylor's Theorem for Analytic Functions of Operators
}

\author{
Wesley E. Brittin and Walter Wyss \\ Department of Physics and Astrophysics, University of Colorado, Boulder, Colorado 80309, USA
}

\begin{abstract}
We discuss analytic functions on a Banach algebra into itself. In particular expressions for derivatives are given as well as convergent Taylor expansions.
\end{abstract}

\section{Introduction}

The problem of expansion of functions of non-commuting operators occurs in many branches of theoretical physics. Many formal schemes [1-5] have been used, but in very few cases [5] has convergence been established. We discuss a case for which convergence is established. Our approach follows in spirit the work [5] of Araki.

\section{Analytic Functions of Operators and Derivatives}

Let $F: \mathbb{C} \rightarrow \mathbb{C}$ be an analytic function in $G=\{z|| z \mid<\varrho\}$. In the domain $G, F$ has a convergent power series expansion

$$
F(z)=\sum_{n=0}^{\infty} c_{n} z^{n} .
$$

The $n^{\text {th }}$ derivative $D^{n} F$ of $F$ also has a convergent power series having the same domain of convergence as $F$.

Let $\mathscr{B}$ be a Banach algebra and denote by $\mathscr{L}=\mathscr{L}^{1}(\mathscr{B})$ the Banach algebra of bounded linear maps $L$ of $\mathscr{B}$ into itself. The norm of $\mathscr{L}^{1}(\mathscr{B})$ is defined by $\|L\|=\sup _{A \in \mathscr{B}} \frac{\|L A\|}{\|A\|}$. We then define the Banach algebras $\mathscr{L}^{n}(\mathscr{B})$ iteratively by $\mathscr{L}^{1}\left(\mathscr{L}^{n-1}(\mathscr{B})\right)$.

Definition 1. Let $\mathscr{B}$ be a Banach algebra and $A, B \in \mathscr{B}$. For $0 \leqq \lambda \leqq 1$ let $A_{\lambda}$ be the linear map from $\mathscr{B}$ into $\mathscr{B}$ defined by

$$
A_{\lambda} B=A B-\lambda d_{A} B \text {, }
$$


with

$$
d_{A} B=[A, B]=A B-B A .
$$

Lemma 1. Let $A_{\lambda}$ be defined as above. Then $\left\|A_{\lambda}\right\| \leqq\|A\|$.

Proof.

$$
\begin{aligned}
\left\|A_{\lambda}\right\| & =\sup _{B \in \mathscr{B}} \frac{\left\|A_{\lambda} B\right\|}{\|B\|}=\sup _{B \in \mathscr{B}} \frac{\|(1-\lambda) A B+\lambda B A\|}{\|B\|} \\
& \leqq(1-\lambda)\|A\|+\lambda\|A\|=\|A\| .
\end{aligned}
$$

Lemma 2. For $A \in \mathscr{B}$ and $n$ a positive integer, the following relations hold in $\mathscr{L}(\mathscr{B})$

$$
\text { 1) } \begin{aligned}
& A^{n}-\left(A-d_{A}\right)^{n}=n \int_{0}^{1} d \lambda A_{\lambda}^{n-1} d_{A}, \\
& \text { 2) } \quad\left(A-d_{A}\right)^{n}=\int_{0}^{1} d \lambda A_{\lambda}^{n}-n \int_{0}^{1} d \lambda \lambda A_{\lambda}^{n-1} d_{A}, \\
& \text { 3) } \quad A^{n}=\int_{0}^{1} d \lambda A_{\lambda}^{n}+n \int_{0}^{1} d \lambda(1-\lambda) A_{\lambda}^{n-1} d_{A} .
\end{aligned}
$$

Proof. First note that $A d_{A}=d_{A} A$ as maps in $\mathscr{L}(\mathscr{B})$. Then

$$
\text { 1) } n \int_{0}^{1} d \lambda A_{\lambda}^{n-1} d_{A}=-\int_{0}^{1} d \lambda \frac{d}{d \lambda} A_{\lambda}^{n}=A_{0}^{n}-A_{1}^{n}=A^{n}-\left(A-d_{A}\right)^{n} \text {. }
$$

2) By partial integration we have

$$
\begin{aligned}
\int_{0}^{1} d \lambda A_{\lambda}^{n} & =\left.\lambda A_{\lambda}^{n}\right|_{0} ^{1}+\int_{0}^{1} d \lambda \lambda n A_{\lambda}^{n-1} d_{A} \\
& =\left(A-d_{A}\right)^{n}+n \int^{1} d \lambda \lambda A_{\lambda}^{n-1} d_{A} .
\end{aligned}
$$

3) Combine 1) and 2).

Lemma 3. $A^{n}-\left(A-d_{A}\right)^{n}=d_{A^{n}}$,

or, equivalently

$$
\left(A-d_{A}\right)^{n} B=B A^{n} .
$$

Proof. The lemma is true for $n=1$. By induction we then find that

$$
\begin{array}{rl}
A^{n+1} & B-\left(A-d_{A}\right)^{n+1} B \\
& =A^{n+1} B-\left(A-d_{A}\right) B A^{n} \\
& =A^{n+1} B-A B A^{n}+\left[A, B A^{n}\right] \\
& =A^{n+1} B-B A^{n+1}=d_{A^{n+1}} B .
\end{array}
$$


An analytic function $F$ with radius of cenvergence $\varrho$ gives rise to a map $F: \mathscr{B} \rightarrow \mathscr{B}$

by means of

$$
F(A)=\sum_{n=0}^{\infty} c_{n} A^{n}
$$

This map is defined for all $A \in \mathscr{B}$ for which $\|A\|<\varrho$.

Lemma 4. For $F$ analytic and $\|A\|<\varrho$ we have

$$
F(A)-F\left(A-d_{A}\right)=d_{F(A)},
$$

or, equivalently for $B \in \mathscr{B}$,

$$
F\left(A-d_{A}\right) B=B F(A) .
$$

Proof. This follows from Lemma 3 and the fact that $\left\|A-d_{A}\right\| \leqq\|A\|<\varrho$.

It may be noted that for $F=\exp$ we recover the well-known result

$$
\exp \left(-d_{A}\right) \cdot B=\exp (-A) B \exp (A) \text {. }
$$

Let $A(t) \in \mathscr{B}$ be a differentiable path in $\mathscr{B}$ for $t \in I \subset R$ and for which $\|A(t)\|<\varrho$ and $\frac{d}{d t} A(t) \in \mathscr{B}, \forall t \in I$. Then $F(A(t))$ is a $\mathscr{B}$-valued function of $t$.

Theorem 1. The function $F(A(t))$ is differentiable and its derivative is given by

$$
\frac{d}{d t} F(A(t))=\int_{0}^{1} d \lambda D F\left(A_{\lambda}\right)\left(\frac{d A}{d t}\right) .
$$

Proof. It suffices to prove the statement for powers of $A(t)$, i.e. to show that

$$
\frac{d}{d t} A(t)^{n}=\int_{0}^{1} d \lambda n A_{\lambda}^{n-1}\left(\frac{d A}{d t}\right) .
$$

The statement is clearly valid for $n=1$, and by induction

$$
\begin{aligned}
\frac{d}{d t} A^{n+1} & =A^{n} \frac{d A}{d t}+\frac{d A^{n}}{d t} A \\
& =A^{n} \frac{d A}{d t}+\left\{n \int_{0}^{1} d \lambda A_{\lambda}^{n-1}\left(\frac{d A}{d t}\right)\right\} A \\
& =A^{n} \frac{d A}{d t}+n \int_{0}^{1} d \lambda A_{\lambda}^{n-1}\left(\frac{d A}{d t} A\right) \\
& =A^{n} \frac{d A}{d t}+n \int_{0}^{1} d \lambda A_{\lambda}^{n-1}\left(A-d_{A}\right)\left(\frac{d A}{d t}\right) .
\end{aligned}
$$


With the help of Equation (4) and Equation (5), the above expression becomes

$$
\begin{aligned}
\frac{d}{d t} A^{n+1}= & \left(A-d_{A}\right)^{n}\left(\frac{d A}{d t}\right)+n \int_{0}^{1} d \lambda A_{\lambda}^{n-1} A\left(\frac{d A}{d t}\right) \\
= & \int_{0}^{1} d \lambda A_{\lambda}^{n}\left(\frac{d A}{d t}\right)-n \int_{0}^{1} d \lambda \lambda A_{\lambda}^{n-1} d_{A}\left(\frac{d A}{d t}\right) \\
& +n \int_{0}^{1} d \lambda A_{\lambda}^{n-1} A\left(\frac{d A}{d t}\right) \\
= & \int_{0}^{1} d \lambda\left\{A_{\lambda}^{n}+n A_{\lambda}^{n-1}\left(A-\lambda d_{A}\right)\right\}\left(\frac{d A}{d t}\right) \\
= & (n+1) \int_{0}^{1} d \lambda A_{\lambda}^{n}\left(\frac{d A}{d t}\right) .
\end{aligned}
$$

For an analytic function $F$ we obtain Equation (13), since for $\|A(t)\|<\varrho$ and $0 \leqq \lambda \leqq 1$ we have $\left\|A_{\lambda}(t)\right\| \leqq\|A(t)\|<\varrho$, and hence absolute convergence of the respective power series.

Corollary 1. For the function $F(A(t))$ of Theorem 1 with $A(t)$ twice differentiable and $\frac{d^{2} A(t)}{d t^{2}} \in \mathscr{B}$, we have

$$
\begin{aligned}
\frac{d^{2} F}{d t^{2}}(A(t))= & \int_{0}^{1} d \lambda D F\left(A_{\lambda}\right)\left(\frac{d^{2} A}{d t^{2}}\right) \\
& +\int_{0}^{1} d \lambda_{1} \int_{0}^{1} d \lambda_{2} D^{2} F\left(A_{\lambda_{1}, \lambda_{2}}\right)\left(\frac{d A_{\lambda_{1}}}{d t}, \frac{d A}{d t}\right)
\end{aligned}
$$

where it is implied that $D^{2} F\left(A_{\lambda_{1}, \lambda_{2}}\right)$ acts on $\frac{d A_{\lambda_{1}}}{d t}$ and the result of the $\lambda_{2}$-integration acts then on $\frac{d A}{d t}$.

Proof. We have only to note that $\left\|A_{\lambda_{1}, \lambda_{2}}\right\|<\varrho$ and $\int_{0}^{1} d \lambda_{2} D^{2} F\left(A_{\lambda_{1}, \lambda_{2}}\right) \in \mathscr{L}^{2}(\mathscr{B})$. This map is applied to $\frac{d A_{\lambda_{1}}}{d t}$ and yields a result in $\mathscr{L}^{1}(\mathscr{B})$, which after $\lambda_{1}$-integration acts on $\frac{d A}{d t}$.

We now obtain a relation between the commutator of an analytic function $F(A)$ with an element of $\mathscr{B}$ and the commutator of $A$ with the same element.

\section{Lemma 5. 1}

$$
d_{F(A)}=\int_{0}^{1} d \lambda D F\left(A_{\lambda}\right) d_{A} .
$$

Proof. The extension of Lemma 2 to analytic functions yields

$$
d_{F(A)}=F(A)-F\left(A-d_{A}\right)=\int_{0}^{1} d \lambda D F\left(A_{\lambda}\right) d_{A} .
$$


It may be noted that if we define the map

$$
\frac{d_{F(A)}}{d_{A}}=\int_{0}^{1} d \lambda D F\left(A_{\lambda}\right),
$$

then the expression for the derivative [Eq. (13)] takes on the appearance of the chain-rule of elementary calculus, i.e.

$$
\frac{d F(A)}{d t}=\frac{d_{F(A)}}{d_{A}} \frac{d A}{d t} .
$$

Corollary 2. For the special case that the tangent to the path $A(t)$ admits the following representation

$$
\frac{d A}{d t}=d_{A} H, \quad H \in \mathscr{B} .
$$

We obtain the Heisenberg equation

$$
\frac{d}{d t} F(A(t))=[F(A(t)), H]
$$

Proof.

$$
\begin{aligned}
\frac{d}{d t} F(A(t)) & =\int_{0}^{1} d \lambda D F\left(A_{\lambda}\right) d_{A} H \\
& =d_{F(A)} H=[F(A(t)), H]
\end{aligned}
$$

by Lemma 5 .

\section{Taylor's Theorem for Analytic Functions}

We now apply our results to find the Taylor expansion of $F(A+\lambda B)$ in powers of $\lambda$.

\section{Theorem 2.}

$$
\begin{aligned}
F(A+\lambda B)= & \sum_{n=0}^{\infty} \frac{\lambda^{n}}{n !} \int_{0}^{1} d \lambda_{1} \ldots \int_{0}^{1} d \lambda_{n} D^{n} F\left(A_{\lambda_{1} \lambda_{2} \ldots \lambda_{n}}\right) \\
& \cdot\left(B_{\lambda_{1} \ldots \lambda_{n-1}}, B_{\lambda_{1} \ldots \lambda_{n-2}}, \ldots, B_{\lambda_{1}}, B\right)
\end{aligned}
$$

with non-zero radius of convergence for $\|A+\lambda B\|<\varrho$.

Proof. Let $X(\lambda)=A+\lambda B$ then for $\|A+\lambda B\|<\varrho$, and because of Theorem 1 and the fact that $\frac{d^{n} X(\lambda)}{d \lambda^{n}}=0, n \geqq 2$, the required higher derivatives can be obtained. Convergence is guaranteed from the facts that $\left\|A_{\lambda_{1} \ldots \lambda_{n}}\right\| \leqq\|A\|,\left\|B_{\lambda_{1} \ldots \lambda_{n}}\right\| \leqq\|B\|$, and that $\exists R>0$ such that $F\left(z_{1}+\lambda z_{2}\right)$ converges absolutely for $\left|z_{1}+\lambda z_{2}\right|<\varrho$ and $|\lambda|<R$.

Formula 18 when applied to the function $\exp \{-i t(H+\lambda V)\}$ yields the Feynman-Dyson series. 


\section{References}

1. Magnus, W.: Comm. Pure Appl. Math. 7, 649 (1954)

2. Wilcox, R. M.: J. Math. Phys. 8, 962 (1967)

3. Guenin, M.: Helv. Phys. Acta 41, 439 (1968)

4. Bialyniki-Birula, I., Mielnick, B., Plebanski, J.: Ann. Phys. 51, 187 (1969)

5. Araki, H.: Ann. Scient. Ec. Norm. Sup. 6, 67 (1973)

Communicated by H. Araki

Received February 23, 1976 